\title{
Comparative Search of Entities
}

\author{
Abhijeet Ramesh Thakare* and Parag S. Deshpande ${ }^{\dagger}$ \\ Department of CSE, National Institute of Technology, \\ Nagpur, Maharashtra 440010, India \\ *abhijeet.thakare@gmail.com \\ †psdeshpande@cse.vnit.ac.in
}

Received 26 March 2017

Revised 27 June 2017

Accepted 22 July 2017

\begin{abstract}
Nowadays, every manufacturer or retailer displays their product information on various websites. The customer has to visit, the number of such web pages to choose the right product, because the information is not available at one place. There are some websites that show such information in one place, but they are product specific and in general information is manually updated. In this paper, we propose a novel concept of web-spreadsheet, which displays product information by crawling through related web pages and generates information like a spreadsheet where each row represents product information and each column represents product attributes. We are extracting the product name of specified product class using decision tree-based classifier by features obtained using Part of Speech (POS) tagging and distance measure. It also extracts the value-measure pairs of preset attributes using distance measure, POS tagging and Data type. This approach will save a lot of time of comparing different products and customers need not have to scan a number of websites for comparison. We present promising results in various product classes which surpass many existing techniques in the literature. The proposed method can work accurately without initial trained labeled data which is expensive to obtain.
\end{abstract}

Keywords: Entity extraction; attribute value extraction; product comparison; web spreadsheet.

\section{Introduction}

Fastest growing e-commerce world has provided tremendous opportunities to the customers for purchasing products from different manufacturer or retailers site, such as Amazon.com, Flipkart.com. However, the features and the price of the product are biased towards one site. Single website provides a limited amount of information regarding features (attributes) of the product. For a product, prices

This is an Open Access article published by World Scientific Publishing Company. It is distributed under the terms of the Creative Commons Attribution 4.0 (CC-BY) License. Further distribution of this work is permitted, provided the original work is properly cited. 
also may vary from one website to another. Customer will find various values of the features (attributes) as well as prices for a particular product on different retailer sites. Therefore, the customer has to visit various retailer's site to choose the right product.

For a specific product, some websites display the complete information, but they lack in showing the information for other products. In future, if any, change to the product information occurs, information is manually updated by them. There are some websites, which provides the facility to compare the similar class products. The customers pick the products of their choices, compare the features and select the most suitable ones. In this comparison process, generally all the features are taken into consideration. Some customers are mainly interested in specific attributes of the product before purchasing it, which is unhandled in the current comparison process.

A web-spreadsheet view will satisfy the customer and handles all the above discussed scenarios. Hence, in the proposed research, the authors focus on a novel search problem for comparative search of entities (CSE). The main objective of CSE is to extract relevant and attribute level information related to entities along with the entity (product) name in the form of a spreadsheet. Attributes, in which customers are interested, are chosen in advance which will form the columns of the spreadsheet. Product information, i.e. values (consisting of value-measure pair) are extracted efficiently by crawling through related web pages. This approach provides all information (value) for a particular products attribute.

CSE is inspired by Relational Aggregated Search (RAS) which is a new information retrieval area emerging nowadays. In RAS, information is extracted and aggregated in the form of small chunks using their relations. RAS consists of three main components, namely Query Dispatching, Relation Retrieval and Result Aggregation [1]. In the proposed work, authors mainly focus on Relation Retrieval and Result Aggregation operation. In Relation Retrieval, authors targeted on instanceclass relation to retrieve complete entity name and instance-attribute relation to extract value-measure pair given predefined attribute and its data type. For example, if a user submits a query for the product class Tablet with attributes such as Price, Display, Camera and Memory then the entities belongs to that product class and attribute values of those entities are retrieved.

This approach would not only reduce the effort of user by crawling through each hyperlink for finding relevant information, but also able to deliver fine grained attribute-value information.

Gathering the information in a consolidated spreadsheet is having following challenges.

- Each webpage consists of descriptions of particular entity, which is stored in a much unstructured manner. The entity name may appear on a webpage in ambiguous form. For example, when user intention is electronic tablet, it may search webpage of medicinal tablet.

- On the web page, the attribute value pair may not be directly specified, so correct value of the attribute has to be extracted. 
Considering above challenges, the research work is carried out and the major contributions of it, is summarized as follows:

\subsection{Contributions}

- A novel complete entity name extraction process is proposed to extract complete entity name from unstructured text.

- A novel value-measure extraction method is designed to extract value-measure pair of the specified attribute and its data types and which works independently of sequencing of attribute and its value.

- Extraction of adjective type values whose attribute is noun are fetched using unique string type value extraction methodology using the Word Net.

- Generating web-spreadsheet dynamically without referring any additional information and irrespective of domain without targeting some specific set of retailer websites.

- To our best knowledge, no other system is designed which extracts entities of specific product class, value-measure pairs for a given preset attributes as we have addressed in this paper. Our web crawler crawls almost all related web pages to efficiently extract entities and value-measure pairs.

- For entity and attribute-value extraction, the proposed method achieves very good accuracy which is measured in terms of precision, recall and F1-measure that is comparable to prominent entity and attribute value extraction methods discussed in the literature.

\section{Related Work}

Various techniques illustrate the extracting information from unstructured documents that are natural language documents [2,3]. A template independent wrapper has been developed which exercises labeled news pages from a single site [4]. This wrapper has been used to extract plain texts of news embedded in news article body. The relation between news article and news body is also explored by developing novel features for them. Geographical coordinates and addresses of various organizations from unstructured web sites of organizations are extracted in [5].

Some techniques also deal with semistructured documents [6]. These documents are based on HTML, in which information is stored in different structures like forms, lists, tables. Semantics from semistructured data from web documents using ORA-SS (object- relationship- attribute model) are efficiently extracted [7]. ORA-SS is used to facilitate designing of semistructured databases. A technique [8] of materials knowledge extraction from HTML tables which is based on sibling comparison has been addressed.

Many named entity extraction technique has been proposed in the last few years and still today, named entity extraction is challenging problem because, entities are 
scattered over web pages in unstructured and ambiguous manner. Extracting entity (instance) from web pages by identifying instances-class relation is also demanding.

Work presented by [9], addresses domain independent unsupervised named entity extraction method. Using predicates and patterns, named entities are extracted from web corpus. In this work, a set of rules is created using Bootstrapping, which uses domain independent extraction patterns. These rules are applied to extract the information from web pages. Based on mutual information between extracted instances of a class and set of discriminator associated with that class, the accuracy is calculated.

Named entity recognition technique using undirected graphical models called as Conditional Random Fields (CRFs) has been depicted [10]. Automated feature induction method has been used in this work. Initially, atomic features are created by the user. As compared to traditional approaches accuracy is improved. It also reduces feature count. Experimentation has been performed on CONLL-2003 named entity shared task dataset.

Focus on context patterns for extracting named entity from unlabeled data has been worked out [11]. In this work, language independent technique is used. The initial seed list is created and it is expanded gradually by searching more instances for matching context patterns from unlabeled data.

Support Vector Machine (SVM)-based parser has been proposed [12]. Using statistical techniques for an input sequence $n$-best words are calculated with the features based on their character position and the type. Finally, SVM is used to aggregate character into words and named entity is identified. This work uses CRL NE data for evaluation.

Many researchers have done work in the past few years on extracting features in terms of attributes and their values from web pages. Recent work on extracting pairs of comparative sentences from online reviews of products has been discussed [13]. In this work, features are extracted from pros and cons sentences of the customer views of the product. Using POS tagging, frequent occurrence of noun and noun phrases are treated as features. Frequent feature identification from online reviews is focused [14]. In this work, opinion words are identified initially. Then, semantic orientations (positive or negative) of opinion words are determined using bootstrapping technique, which is based on Word Net. Point of mutual information between noun phrases and related context pattern for extracting product features and opinions from reviews has ben calculated [15]. Work done in [16], targets on mining frequent features based on association rule mining technique and natural language processing methods.

Technique for extracting attribute-value pairs from textual product descriptions for implicit and explicit attributes has been addressed [17]. Implicit attributes are semantic attributes which are implicitly mentioned in the product description. While, explicit attributes are physical attributes. Experiments were performed on extracting explicit attributes using Naïve Bayes as a supervised learning and Co-training Expectation Maximization (Co-EM) as a Multiview semi-supervised learning algorithm. Sporting goods category website is used for extracting attribute values. 
Work presented in [18], illustrates extracting numerical and textual attributevalue pairs on three variations such as Tweets, Web-Links and Tweets+ Web-Links for 15 events. For extracting numerical attribute-value pairs, splitting of sentences based on a set of delimiters is done. Next, some special cases are handled such as, extracting attribute-value pairs which are mentioned side by side or in a sequence using common noun or proper noun. In this, the word is marked as attribute if it is a noun and coming next to the word. Finally, the remaining sentences (which does not consist of special cases) are handled using dependencies (subject and object concept). Textual attribute-value pairs are extracted based on the relationship with the subject or root word of tweet or preposition dependencies.

Extracting attribute-value pairs using automatically induced Knowledge Base (KB) for a particular category has been demonstrated [19]. In this method, KB is automatically created from tables and other structures which are available in unstructured web pages. Using KB, web pages are annotated automatically. This annotated corpus is used to train the model for extraction. Some attribute value pairs are not present in this model because, annotated corpus is not created manually. Therefore, this extraction model lacks in extracting some attribute value pairs from unstructured test data.

\section{Proposed Approach}

The proposed approach is described using following sections. These sections give the meaning of definitions and terms used in the Algorithm 1, major steps of the algorithm and explanation of each step of the algorithm.

\subsection{Definitions and terms}

Class Name (CLNAME): CLNAME is a product class name which is provided by the user. For example, value of CLNAME can be Tablet, Mobile-Phone, and MixerGrinder etc. Final objective is to generate a spreadsheet for class CLNAME.

Web Spreadsheet $(S P): S P$ is a spreadsheet which consist of $\left\{T_{1}, T_{2}, T_{3}, \ldots, T_{n}\right\}$ rows. Consider, $D$ be a set of web pages related to product class, where, $D=\left\{D_{0}, D_{1}\right.$, $\left.D_{2}, \ldots, D_{n}\right\}$ and $n \geq 0$. Algorithm 1 , crawls over each $D_{i}$ one by one and generates one row $T_{i}$ for spreadsheet $S P$. We define function $f: \mathbb{D} \rightarrow \mathbb{T}$ where, $f$ is injective and onto.

Web Spreadsheet Columns $(C L)$ : Let, $A T$ consists an array of elements $\left(\left\{A_{1}, D T_{1}\right\}\right.$, $\left.\left\{A_{2}, D T_{2}\right\} \ldots\left\{A_{n}, D T_{n}\right\}\right)$. Every element of AT consists of an attribute and its data type. These attributes, i.e. $A_{1}, A_{2}, \ldots, A_{n}$ and data types $D T_{1}, D T_{2}, \ldots, D T_{n}$ are user defined. Attributes $A_{1}, A_{2}, \ldots, A_{n}$ will be columns of a spreadsheet where, $n \geq 1$.

Web Spreadsheet Row $\left(T_{i}\right)$ : It is one of the row of a spreadsheet. $T_{i}$ consists of $\left(E_{i}, I A_{i 1}, I A_{i 2}, \ldots, I A_{i n}\right)$ where, $E_{i}$ is a complete entity name and $I A_{i 1}, I A_{i 2}, \ldots, I A_{\text {in }}$ are value-measure information for attributes $A_{1}, A_{2}, \ldots, A_{n}$ respectively corresponding to document $D_{i}$ where, $n, i \geq 1$. 


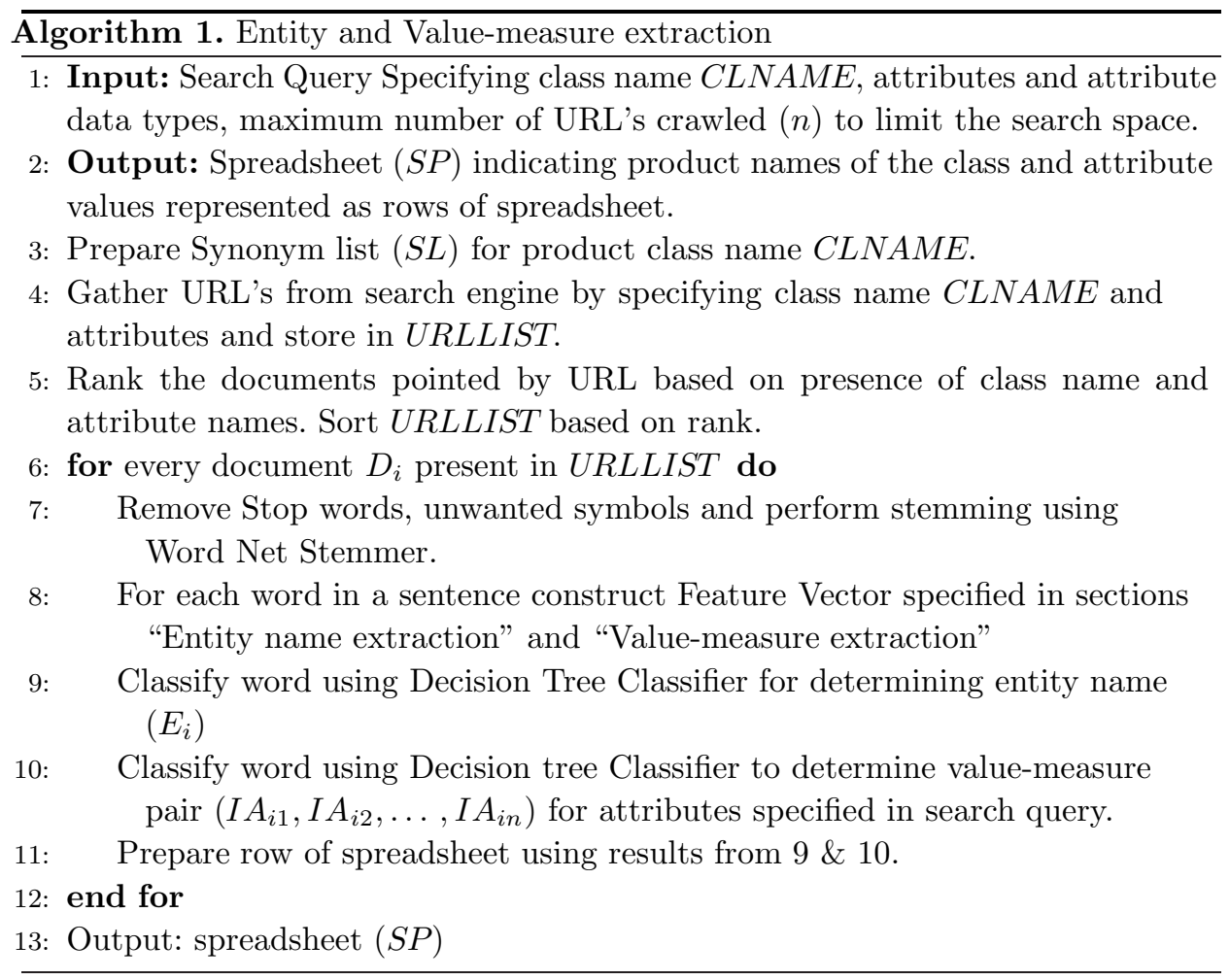

POS tag of a word $W_{i}$ (POS_Tag): POS tag of word $W_{i}$ is defined as POS_Tag. POS tag of word is used to categorize the word as nouns, adjectives, verbs, adverbs etc. Following POS tags are used in the proposed work:

CD — Cardinal Number, NN — Noun, singular or mass, NNP - Proper noun, singular

Synonym List $(S L)$ : Synonym list for class, CLNAME consists of set synonym words of $C L N A M E$. $S L$ is represented as $S W_{1}, S W_{2}, \ldots, S W_{n}$ where, $n \geq 1$.

URLLIST: List to store all related URL, returned by search engine.

\subsection{Input}

Input to the Algorithm is search query mentioning product class name (CLNAME), attribute names $\left(A_{i}\right)$, attribute data types $\left(D T_{i}\right)$ and maximum number of URLs $(n)$ which can be crawled to limit the search space. The attribute which is mentioned earlier in the specification is considered to have high priority. For example, to prepare a spreadsheet for tablet, user can specify product class name as Tablet, attributes as Price, Touchscreen, Camera, Processor and RAM. 
In the proposed work, the authors define following four data types (value-measure) for the attributes: (A) Alphanumeric (B) Numeric-Alpha (C) String (D) Numeric which is explained as follows:

Alphanumeric $(A N)$ : If the word consists of at least one alphabetic letter $(\mathrm{A}-\mathrm{Z}$ or $\mathrm{a}-\mathrm{z})$ and at least one digit (0-9) or combination of digit (0-9) and alphabetic letters then the word falls under alphanumeric category. For e.g. if we take camera as an attribute and its value as $13 \mathrm{MP}$ then type of value of the camera is alphanumeric.

Numeric-Alpha $(N A)$ : Numeric-Alpha category is a subset of alphanumeric category. The only difference is value and measure is separated by one or more spaces. i.e. Number/s-Space-Alphabet/s (String). For e.g. 1 MP.

String $(S T)$ : If a word is formed using alphabetic letters $\mathrm{A}-\mathrm{Z}$ or a-Z or combination of both then, word falls under string category. For example large, big, small and little.

Numeric $(N U)$ : It consists of pure numbers either in float or as an integer. For e.g. 13, 13.4, etc.

\subsection{Preparation of synonym list}

We also maintain $S L$ for product class name where all the synonym words for product class name are stored. $S L$ for class $C L N A M E$ is created using Word Net. $S L$ plays a major role in extracting instance-class relation. The main objective of creating a $S L$ is to target only those sentences of a web page, which contains at least one of the synonym words from list $S L$.

Every Retailer describes their products in a different fashion. They also use different words corresponding to class name $C L N A M E$ to describe the complete name of the product. For e.g. Fig. 1 shows the initial part of product description page for Lenovo Tablet. In this, complete name (Lenovo Tab 2 A8) in the heading part comprises word Tab while in Fig. 2, the complete name (UBI Slate 7CZ Tablet) consists of word Tablet.

While describing the product, retailers use different substring words. These substring words which are basically a part of a complete entity name. For e.g. Fig. 3 shows the part of product description page for the Samsung Galaxy Tablet. The Complete Name of the product is "Samsung Galaxy Tab 4". The product is described using $n$ number of sentences.

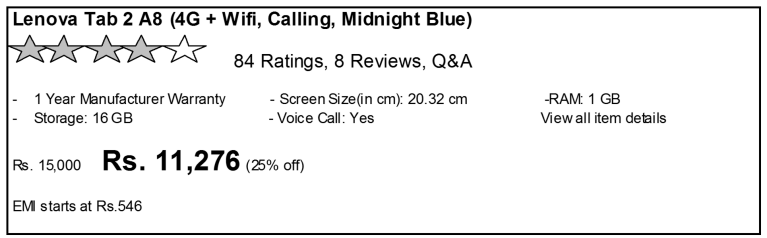

Fig. 1. Initial part of product description page for Lenovo Tablet. 


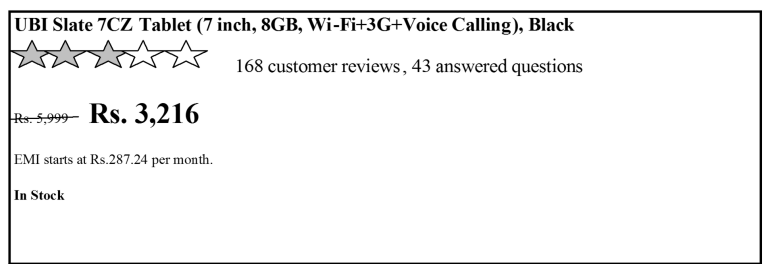

Fig. 2. Initial part of product description page for UBI Slate Tablet.

Packed with host of features, Samsung Galaxy Tab 4 T231 tablet is user friendly budget device. It comes with a large $17.78 \mathrm{cms}$ (7) WXGA multi-touch display on which you can view images and videos with a resolution of $1280 * 800$ pixels. With 3 megapixel rear camera of this tablet, you can capture stunning shots. It comes with zoom features that less you bring your subject closer to the camera lens. You can take amazing selfies with its 1.3 megapixels front camera. Equipped with $1.2 \mathrm{GHz}$ quad-core processor, it offers responsive navigation across different applications. Android operating system and 1.5 GB RAM boosts the performance of this Galaxy tablet. On buying this Samsung Galaxy tablet, you not only get lowest price guarantee but also 1 Year Samsung India warranty on the product.

Fig. 3. Product description page for Samsung Galaxy Tablet.

Consider the two sample sentences described as follows:

Sentence 1: "Packed with a host of features, Samsung Galaxy Tab 4 T231 Tablet is a user friendly budget device."

Sentence 2: "Android operating system and 1.5 GB RAM boosts the performance of this Galaxy Tablet."

In sentence 1 complete entity name, i.e. "Samsung Galaxy Tab 4 T231" is used while in sentence 2, substring word Galaxy is used. In sentence 1, Tablet and Tab are used as a synonym words while in sentence 2 Tablet is used as a synonym word.

\subsection{URL extraction module}

Using jsoup 1.8.2 Java library, each URL in the search result which is returned by search query is accessed, parsed and decoded. All child URLs from each search result URL are also extracted, parsed and decoded. Iteration through every URL page and their child URL pages are done and the complete decoded URLs for these pages are stored in a database table. To ensure that, the URL is not visited twice, the presence of a URL in the database table is checked before storing it. If it is not present in the database table, then the URL is stored into the table as well as in list URLLIST. Now, the database table as well as list URLLIST will consist of unique URLs. These URLs will be given as input to the module Data Preprocessing. 


\subsection{Ranking of documents}

The extracted documents are ranked based on presence of product class name and specified attributes. If more attributes are present then, the rank of the document is higher. If the same number of attributes are present, then document which is having more priority attributes are ranked higher.

\subsection{Data preprocessing}

In data preprocessing all special symbols, stop words are removed.

\subsection{Entity name extraction}

\subsubsection{Building a classifier using J48 decision tree [Weka] for entity}

Classifier is designed by generating feature vector for each word, preparing data for training and using decision tree for classification.

\subsubsection{Construction of features vector for each word}

Each document, if it is not present in the database table, then, the URL is stored into the table as well as in list $D_{i}$ is crawled one by one whos URL is already stored in the list URLLIST as mentioned in URL extraction module. Feature vector (Training data) is prepared using Tablet, Washing Machine and Tennis Racket classes by crawling the related website URL of different retailers. Every word in document $D_{i}$ is represented as feature vector which is shown in Table 1.

Information in Table 1, depicts the particular words Document id, sentence number, the position of the word in a sentence, its POS tag and Label. Label attribute takes two possible values either YES or NO for entity classifier. YES means particular word is an entity or an instance of the respective class otherwise it is labeled as NO. Position of word is calculated with respect to Class Name Word $(C L N A M E)$ or its synonym word, i.e. CLNAME is given position 0 and first left word is given position -1 and the position is decreased by -1 as we move left side of the sentence. First right word is given position 1 and the position is increased by 1 as we move the right side of the sentence.

Based on the sentence number of the word and position within the sentence, above training data is discretized, which is shown in Table 2.

Table 1. Feature vector representation (entity).

\begin{tabular}{llllll}
\hline DocId & Attribute word & Sentence_No & Position & POS_Tag & Label \\
\hline
\end{tabular}

Table 2. Discretized feature vector representation (entity).

\begin{tabular}{lllll}
\hline DocId & Attribute word & POS_Tag & Section & Label \\
\hline
\end{tabular}


Here, a new attribute section is introduced which is dependent on Sentence_No and Position attribute as follows:

Case 1. If Sentence_No $<\alpha$ and Position $>-\beta$ and Position $<\gamma$ then Section is Nearer.

Case2. If Sentence_No $>\alpha$ and Position $<-\beta$ OR Position $>\gamma$ then Section is Far.

The main purpose of introduction of Section attribute is, to define initial part of the web page. While describing the product on a web page, heading (which consists of the complete name of the product) is placed in the initial part of a web page so, authors concentrated on those sentences placed in the initial part. Here, authors target on the initial part of webpage because, the first occurrence of entity name occurs in the initial part of the web page. A section which comes under initial part is defined as Nearer otherwise the section is Far.

\subsubsection{Labeling training data}

Those words which belong to instance of product class name in the training data is labeled as either YES or NO. Labeling is done as follows:

Case 1. If POS_Tag $=$ NN or NNP and Section $=$ Nearer then Label is YES.

Case 2. If POS_Tag $!=$ NN or NNP and Section = Nearer or Far then Label is NO.

Here, we have considered a sufficient amount of features to label the word as an entity. Features such as POS_Tag of a words which are present before and after synonym word, distance of the words with respect to synonym word and Sentence number in which the word appears are taken into consideration.

\subsubsection{Extracting the entity name using $J_{4} 8$ classifier}

For deciding the status of words in the test documents decision tree classifier is used. The decision tree is trained using training data generated from a variety of product classes. Words in the test documents are fed to a classifier to decide the status of the words. The word which is classified as a product class instance forms the entity name of the product class and it will constitute the row label in the spread sheet.

\subsection{Value-measure extraction}

To decide words for attribute value, classifier is designed by generating feature vector of each word, preparing data for training and using decision tree for classification.

\subsubsection{Building a classifier using $J 48$ decision tree for the value-measure}

Classifier is designed by generating feature vector for each word, preparing data for training and using decision tree for classification. 
For deciding the status of the word, we have preferred decision tree classifier over other types of classifier like SVM [20], Neural Networks [21] and Naïve Bayes [22] because of following reasons:

- The feature vector consists of numeric and categorical attributes.

- The classification decision is sometimes influenced only by some set of attributes rather than all attributes, while in other types of classifiers always all the attributes have influenced classification decisions.

- The decision tree classifier utilizes nonparametric technique and can be trained even if data is less, while other techniques require large data to estimate correct probability distributions.

- The other classifier performance depends on algorithm parameters like kernel function (SVM), activation function (NN) and probability distribution function (Naïve Bayes).

J48 Decision Tree classifier is the Java Implementation of a C4.5 algorithm in Weka tool. Algorithm C4.5 [23] is an extension of ID3 algorithm. One of the first algorithms for Decision tree implementation was an ID3 algorithm. The main reason for choosing C4.5 over ID3 is due to following reasons:

- The ID3 algorithm is over sensitive to features having a large number of values, which is not suitable for the Internet Search activity [24]. In this case, C4.5 is best suitable.

- As our feature vector consists of numeric and categorical attributes, C4.5 is best suitable. ID3 does not handle numerical attributes.

- Algorithm ID3 does not handle missing values, while C4.5 is efficient to handle missing values.

- C4.5 allows post pruning of decision trees. This will help to reduce error rates in unseen testing data. ID3 does not support pruning activity.

\subsubsection{Construction of feature vector for each word}

Training data is prepared using Tablet, Washing Machine and Tennis Racket product classes by crawling related websites of different retailers. Every word in Document $D_{i}$ is represented as a feature vector, which is shown in Table 3.

Here, the position of the word is always calculated with reference to the word indicating attribute word. As shown in Table 3, feature word indicates the particular words Document id, sentence number, the position of the word in a sentence, its POS tag and Label. Here DocId, Sentence_No, Position, POS_Tag are independent variable and Label is dependent variable. Label attribute takes two possible values either

Table 3. Feature vector representation (value-measure).

\begin{tabular}{llllll}
\hline DocId & Attribute word & Sentence_No & Position & POS_Tag & Label \\
\hline
\end{tabular}


Table 4. Discretized feature vector representation (value-measure).

\begin{tabular}{lllll}
\hline DocId & Attribute word & Occurrence & Data Type & Label \\
\hline
\end{tabular}

YES or NO. If a particular word is indicating the value of a particular attribute, then it is indicated by YES, otherwise it is indicated as NO. Position of word is calculated with respect to attribute word, i.e. attribute word is given position 0 and first left word is given position -1 and the position is increased by -1 as we move the left side of the sentence. First right word is given position 1 and the position is increased by 1 as we move the right side of the sentence.

Above training data is discretized, which is depicted in Table 4.

Here, a new attribute Occurrence is introduced which is dependent on Position and POS_Tag attribute as follows:

Case 1. If POS_Tag $=\mathrm{CD}$ and word is having the first occurrence of the word either from the left side or right side with reference to attribute word then Occurrence is set as First.

Case 2. If POS_Tag $=$ CD and word is not having first occurrence of the word either from left side or right side with reference to attribute word then Occurrence is set as Later.

Case 3. If POS_Tag! = CD then Occurrence is set Random.

A new attribute Data Type is also introduced which is dependent on POS_Tag and Position attribute and its value is set as follows:

Case 4. If POS_Tag $=\mathrm{CD}$ then Data type is set as $A N$ (Alphanumeric) or $N U$ (Number).

Case 5. If POS_Tag! = CD then Data type is set as $S T$ (String).

Case 6. If Position $=(-\mathrm{X})$ and POS_Tag $=\mathrm{NN}$ or NNP and Position $=(-\mathrm{X}-1)$ and POS_Tag $=\mathrm{CD}$ then Data type is set as $N A$ (Numeric-Alpha).

Case 7. If Position $=(\mathrm{X})$ and POS_Tag $=\mathrm{CD}$ and Position $=(\mathrm{X}+1)$ and POS_Tag $=$ NN or NNP then Data type is set as NA (Numeric-Alpha).

\subsubsection{Labeling training data}

Those words which belong to attribute value of product class name in the training data is labeled as either YES or NO. Labeling is done as follows:

Case 1. If Occurrence $=$ First and Data Type $=A N$ or $N A$ then Label is YES.

Case 2. If Occurrence $!=$ First or Data Type $!=A N$ or $N A$ then Label is NO.

Here, we have considered a sufficient amount of features to label the word as value-measure pair. Features such as an Occurrence of a word which considers distance of the words with respect to attribute word, Data Type of the words which are present before and after attribute word and POS_Tag of the word are taken into account. 


\subsubsection{Extracting value-measure pair using J48 classifier}

For deciding the status of words in the test documents, decision tree classifier is used. The decision tree is trained using training data generated from a variety of product classes. Words in the test documents are fed to a classifier to decide the status of the words. The word which is classified as attribute value of the product class instance forms the column value of the row belongs to that instance of the product class in the spreadsheet.

The accuracy of the classifier for entity extraction and attribute value extraction is measured using precision, recall and F1-measure by N-Fold cross-validation and the results are mentioned in Sec. 5 .

The proposed algorithm gives a solution to deal with different attribute descriptions about the same feature of the product. When the information about the same product is available on different websites, then instead of reporting aggregate information, the algorithm will add one row from each website. Moreover, if different information is available about the same attribute in the same web page then the algorithm tries to produce the best of all such information if it matches with attribute data type. This feature of algorithm enables the user to get more precise information for product comparison. This can be explained as follows:

Consider the following two sentences taken from two different retailers website for a particular mobile phone (Lenovo K3 Note):

Retailer 1: Sentence 1: "Powerful 64-bit 1.7 GHz processor, camera of $13 \mathrm{MP} . "$ Retailer 2: Sentence 2: "The device is powered by a Octa core, $1.7 \mathrm{GHz}$, CortexA53 processor paired with 2 GB of RAM."

Sentences 1 and 2 describe different attribute descriptions for the same feature of the product. Here, feature "processor" is taken into consideration. The proposed algorithm extracts descriptions of an attribute which are at the shortest distance with reference to an attribute word. Consider the "Sentence 1" taken from "Retailer1" website as shown above. After removing stop words and special symbols (as described in Sec. 3.6), sentence 1 will be represented as shown in Table 5.

Our proposed algorithm assigns position 0 for the attribute word "processor". The other words which are at left and right of the attribute word "processor" are assigned positions accordingly as shown in Table 5. As we move left side from the attribute word processor, the position is decreased by 1 and as we move the right side of the attribute word position is increased by 1 .

For Sentence 1, using "Case 1", "Case 2" and "Case 4" from Sec. 3.8.2, valuemeasure pair is extracted for attribute "processor" is "64-bit $1.7 \mathrm{GHz}$ " because,

Table 5. Position array for sentence.

\begin{tabular}{lcccccc}
\hline Position & -3 & -2 & -1 & 0 & 1 & 2 \\
\hline Word & Powerful & 64 -bit & $1.7 \mathrm{GHz}$ & Processor & Camera & $13 \mathrm{MP}$ \\
Data type & $S T$ & $A N$ & $A N$ & $S T$ & $S T$ & $A N$ \\
\hline
\end{tabular}


Table 6. Sample spreadsheet for mobile-phone for "Lenovo K3 Note".

\begin{tabular}{lcccccr}
\hline Entity-name & Price & Camera & RAM & Processor & Touchscreen & Size \\
\hline Lenovo K3 Note & 12,000 & $13 \mathrm{MP}$ & $2 \mathrm{~GB}$ & $\mathbf{6 4}$-bit 1.7 GHz & 5.5 inches & Large \\
Lenovo K3 Note & 11,700 & $13 \mathrm{MP}$ & $2 \mathrm{~GB}$ & $\mathbf{1 . 7} \mathbf{G H z}$ Cortex-A53 & 5.5 inches & Large \\
\hline
\end{tabular}

"1.7 GHz" is shortest distance word (whose Data type is "Alphanumeric") with reference to "processor" word as compared with the word "13 MP" and "64-bit" is also word whose data type is "Alphanumeric" and is immediate prior word of "1.7 GHz".

In Sentence 2, using "Case 6" from Sec. 3.8.2, value-measure pair is extracted for attribute "processor" is " $1.7 \mathrm{GHz}$ Cortex-A53" because, POS_Tag of " $1.7 \mathrm{GHz}$ " is "CD" (Cardinal Number) and POS_Tag of "Cortex-A53" is "NN" (Noun-Plural).

From all the above scenarios, we conclude that, for a particular feature "processor" our algorithm extracts two different attribute descriptions, i.e. "1.7 GHz" and "1.7 GHz Cortex-A53" from two different retailers web pages for a particular product "Lenovo K3 Note" and stores in a web-spreadsheet as shown in Table 6 . Rows 1 and 2 in Table 6 correspond to product information extracted from Retailers 1 and 2 web pages, respectively.

Though the work described in this paper is inspired by RAS, instead of aggregating the information, we are providing all the information so that, the information is not lost and users can perform a better comparison. Here, in our work for a particular product, for e.g. for the product, Lenovo K3 Note, if two different retailers are showing prices on their web pages as 12,000 and 11,700, respectively, then our algorithm extracts the product information (Product Name, Price, Attribute-Value pairs) from two different retailer's web page and store this information as a two different rows in spreadsheet as shown in Table 6.

As discussed earlier, our algorithm extracts different attribute descriptions for the same feature for the particular product from different retailers web pages and stores this information in a web-spreadsheet. The main purpose of our algorithm is not to lose the different attribute description for the same product feature. Another advantage of performing this task is that, it will save a lot of time of the customers for comparing the same product having different prices because, all the product information (corresponding to all the retailers) along with the prices will be available in spreadsheet so, customers will not only able to do a comparison on the basis of prices but also get a consolidated view of the same product feature attribute description as shown in Table 6 .

\section{Experimental Setup}

Experiments were performed on Windows Server 2012 (R2) and softwares used are as follows:

- NetBeans IDE 8.0.2

- Java JDK 1.7.0.80 
- Jsoup1.8.2 API is used, which is used to connect to the web document url and extracts all the sentences.

- Stanford Part Of Speech (POS) tagger v. 2.0 for tagging the terms of web pages.

- Word Net Stemmer for obtaining word stems.

- Java Word Net Interface (JWI) API for accessing Word Net library and obtaining values for an attribute.

\section{Results and Discussions}

\subsection{Building a classifier}

Both the Classifiers for entity and value-measure extraction are trained using class names as Tablet, Washing Machine and Tennis Racket. Effectiveness of algorithm which is described in Proposed Approach section is evaluated by calculating classification accuracy (cross-validation) using $N$-Fold cross-validation and 80-20 split i.e. $80 \%$ data are used for training and $20 \%$ data are used for testing.

\subsubsection{Cross-validation for entity extraction}

Cross-validation is performed on training as well as testing set and results are described in Tables 7 and 8, respectively.

Total Number of URLs (web documents) crawled are 63. Table 7 indicates the accuracy of the model using $N$-Fold cross-validation, where $N=10$. The total of number words extracted are 29,999 out of which 1490 words indicate the entity name and 28,509 words are not an entity.

Using $N$-Fold cross-validation (here, $N=10$ ), we obtain average precision, recall and F1 for both YES and NO label as 98.7\%. A total of 29,6129 words is correctly classified into YES or NO category and 387 words are incorrectly classified.

Accuracy of classifier is also calculated using a 80-20 split technique as depicted in Table 8 . In this method, $80 \%$ of the total number of words is used for Training and $20 \%$ words are used for Testing. Out of 6000 words which are used for testing, 5927

Table 7. Accuracy of classifier built for entity name using 10-fold crossvalidation (on complete training set).

\begin{tabular}{lc}
\hline \multicolumn{2}{c}{10 fold cross-validation } \\
\hline Total number of URLs crawled & 63 \\
Total number of words extracted & 29,999 \\
Total number of words indicating the entity name & 1490 \\
Total number of words indicating not as entity name & 28,509 \\
Correctly classified instances & $29,6129(98.71 \%)$ \\
Incorrectly classified instances & $387(1.29 \%)$ \\
Weighted average precision (for YES and, NO label) & $98.7 \%$ \\
Weighted average recall (for YES and, NO label) & $98.7 \%$ \\
Weighted average F1 (for YES and, NO label) & $98.7 \%$ \\
\hline
\end{tabular}


Table 8. Accuracy of classifier built for entity name using 80-20 split ( $80 \%$ data used for training and $20 \%$ data are used for testing).

\begin{tabular}{lc}
\hline \multicolumn{2}{c}{$80-20$ split } \\
\hline Total number of URLs crawled & 63 \\
Total number of words extracted & 29,999 \\
Total number of words used for training (80\%) & 23,999 \\
Total number of words used for testing (20\%) & 6000 \\
Correctly classified test instances (Either YES or NO) & $5927(98.78 \%)$ \\
Incorrectly classified test instances & $73(1.21 \%)$ \\
Weighted average precision (for YES and, NO label) & $98.8 \%$ \\
Weighted average recall (for YES and, NO label) & $98.8 \%$ \\
Weighted average F1 (for YES and, NO label) & $98.8 \%$ \\
\hline
\end{tabular}

words are correctly classified by the classifier into YES or NO category and 73 words are incorrectly classified. Using this method, we obtained average precision, recall and F1 for both YES and NO label as $98.8 \%$.

\subsubsection{Cross-validation for value-measure pair extraction}

Cross-validation is performed on training as well as testing set and results are described in Tables 9 and 10, respectively.

Total number of URLs (web documents) crawled are 60. Table 9 indicates the accuracy of the model using $N$-Fold cross-validation, where $N=10$. Total of number words extracted are 3000 out of which 210 words indicates value-measure pair and 2790 words are not value-measure pairs. Using $N$-Fold cross-validation (here, $N=10$ ), we obtain average precision, recall and F1 for both YES and NO label as $98.92 \%$. A total of 2967 words is correctly classified into YES or NO category and 33 words are incorrectly classified.

Accuracy of classifier is also calculated using a 80-20 split technique as depicted in Table 10. In this method, $80 \%$ of the total number of words is used for Training and $20 \%$ words are used testing. Out of 3000 words which are used for testing, 594 words are correctly classified by the classifier into YES or NO category and 6 words are

Table 9. Accuracy of classifier built for value-measure pair using 10-fold cross-validation (on complete training set).

\begin{tabular}{lc}
\hline \multicolumn{2}{c}{ 10-fold cross-validation } \\
\hline Total number of URLs crawled & 60 \\
Total number of words extracted & 3000 \\
Total number of words indicating value-measure & 210 \\
Total number of words indicating not as a value-measure & 2790 \\
Correctly classified instances & $2967(98.9 \%)$ \\
Incorrectly classified instances & $33(1.1 \%)$ \\
Weighted average precision (for YES and, NO label) & $98.92 \%$ \\
Weighted average recall (for YES and, NO label) & $98.92 \%$ \\
Weighted average F1 (for YES and, NO label) & $98.92 \%$ \\
\hline
\end{tabular}


Table 10. Accuracy of classifier built for value-measure pair using 80-20 split (80\% data used for training and $20 \%$ data are used for testing).

\begin{tabular}{lc}
\hline \multicolumn{2}{c}{$80-20$ split } \\
\hline Total number of URLs crawled & 60 \\
Total number of words extracted & 3000 \\
Total number of words used for training (80\%) & 2400 \\
Total number of words used for testing (20\%) & 600 \\
Correctly classified test instances (Either YES or NO) & $594(99 \%)$ \\
Incorrectly classified test instances & $6(1.00 \%)$ \\
Weighted average precision (for YES and, NO label) & $99.1 \%$ \\
Weighted average recall (for YES and, NO label) & $99.1 \%$ \\
Weighted average F1 (for YES and, NO label) & $99.1 \%$ \\
\hline
\end{tabular}

incorrectly classified. Using this method, the authors obtained average precision, recall and F1 for both YES and NO label as 99.1\%.

\subsection{Evaluation on different product classes}

The proposed method is used to generate a spreadsheet of product classes of different categories. Accuracy is evaluated using precision, recall and F1 measure. The results of the experimentation of product classes such as Mobile Phone, Mixer Grinder, Television and Electric Timer are described in the following sections.

\subsubsection{Product class name: Mobile-Phone}

Input: Search query specifying class name CLNAME as Mobile-Phone, attributes as Price, Camera, RAM, Processor, Touchscreen and Size, respective data types of the attributes, maximum number of URLs crawled $(n)$ to limit the search space as 20 .

Output: Spreadsheet SP consisting of Price, Camera, RAM, Processor, Touchscreen and Size as Columns and rows of a spreadsheet as their respective value-measure pairs. Sample Spreadsheet SP for Mobile-Phone is shown in Table 11.

As mentioned in Table 12, for a particular attribute, "Total Relevant Doc" column corresponds to the total number of relevant documents having product class name as Mobile Phone and the attribute word. "Relevant Docs Retrieved" indicates actual relevant documents retrieved by the algorithm for a particular attribute or entity name. "Total Doc Retrieved" corresponds to the total number of documents retrieved for attribute or entity name. As mentioned in Table 12, we obtained 302 "Relevant Docs" Retrieved, 332 "Total Doc Retrieved", precision as 0.909, "Total Relevant Doc" as 331, Recall as 0.912 and F1 as 0.910 for entity name. For camera attribute, we obtained 140 "Relevant Docs" Retrieved, 192 "Total Doc Retrieved", precision as 0.729, "Total Relevant Doc" as 329, Recall as 0.425 and F1 as 0.536. Precision, Recall and F1 is calculated as follows:

$$
\text { Precision }=\sum \text { Relevant Doc's Retrieved } / \sum \text { Total Doc Retrieved, }
$$


Table 11. Spreadsheet for mobile-phone.

\begin{tabular}{lcccccr}
\hline Entity-name & Price & Camera & RAM & Processor & Touchscreen & Size \\
\hline Samsung galaxy J7 & 16,900 & 8 MP front & $1.5 \mathrm{~GB}$ RAM & Cortex A53 & 5.5 inches & Large \\
Intex Aqua Q7 & 4599 & $2 \mathrm{MP}$ & $1 \mathrm{~GB}$ & $1.2 \mathrm{GHz}$ & $11.43 \mathrm{~cm}$ & Small \\
Lenovo Vibe K5 & 7499 & $13 \mathrm{MP}$ primary & $2 \mathrm{~GB}$ & 616 octa core & $1280 \times 720$ pixels & Large \\
Apple iPhone 7 & 53,999 & $12 \mathrm{MP}, 7 \mathrm{MP}$ & $2 \mathrm{~GB}$ & M10 Motion & $11.938 \mathrm{~cm}$ & Large \\
& & & Co-processor \\
\hline
\end{tabular}

Table 12. Precision, recall and F1 calculation for mobile-phone class.

\begin{tabular}{lcccccc}
\hline \multicolumn{7}{c}{ Product name: Mobile-phone } \\
\hline $\begin{array}{l}\text { Attribute/ } \\
\text { instance name }\end{array}$ & $\begin{array}{c}\text { Relevant } \\
\text { docs retrieved }\end{array}$ & $\begin{array}{c}\text { Total } \\
\text { doc retrieved }\end{array}$ & Precision & $\begin{array}{c}\text { Total } \\
\text { relevant doc }\end{array}$ & Recall & F1 \\
\hline Entity-name & 302 & 332 & 0.909 & 331 & 0.912 & 0.910 \\
Camera & 140 & 192 & 0.729 & 329 & 0.425 & 0.536 \\
RAM & 152 & 179 & 0.8498 & 318 & 0.477 & 0.610 \\
Processor & 99 & 140 & 0.707 & 225 & 0.44 & 0.545 \\
Touchscreen & 47 & 95 & 0.494 & 122 & 0.385 & 0.432 \\
Size & 37 & 38 & 0.973 & 74 & 0.5 & 0.680 \\
Total & 777 & 976 & & 1399 & & \\
Average & & & 0.7961 & & 0.555 & 0.654 \\
\hline
\end{tabular}

$$
\begin{aligned}
& \text { Recall }=\sum \text { Relevant Doc's Retrieved } / \sum \text { Total Relevant Doc's } \\
& \begin{aligned}
F 1= & (2 * \text { Average_Precision } * \text { Average_Recall }) /(\text { Average_Precision } \\
& + \text { Average_Recall })
\end{aligned}
\end{aligned}
$$

\subsubsection{Product class name: Mixer Grinder}

Input: Search query specifying class name $C L N A M E$ as Mixer Grinder, attributes as Price, Warranty, Capacity, Weight, Height, and Size, respective data types of the attributes, maximum number of URLs crawled $(n)$ to limit the search space as 20 .

Output: SP consisting of Price, Warranty, Capacity, Weight, Height, and Size as Columns and rows of a $S P$ as their respective value-measure pairs. Sample Spreadsheet $S P$ for Mixer Grinder is shown in Table 13.

As mentioned in Table 14, we obtained 320 "Relevant Docs Retrieved", 381 "Total Doc Retrieved", precision as 0.839, "Total Relevant Doc" as 380, Recall as 0.842 and F1 as 0.840 for entity name. For warranty attribute, we obtained 200 Relevant Docs Retrieved, 228 "Total Doc Retrieved", precision as 0.877, "Total Relevant Doc" as 333, Recall as 0.600 and F1 as 0.712 .

\subsubsection{Product class name: Television}

Input: Search query specifying the class name CLNAME as Television attributes as Price, Television Size, Response Time, Weight, Angle and Size, respective data 
Table 13. Spreadsheet for Mixer-Grinder.

\begin{tabular}{lcccccr}
\hline Entity-name & Price & Warranty & Capacity & Weight & Height & Size \\
\hline Maharaja Whiteline & 1899 & 1 Year & 0.7 liter & $4.7 \mathrm{~kg}$ & $16.5 \mathrm{inch}$ & Large \\
Philips HL1645 & 3961 & 2 Years & $0.5 \mathrm{l}$ & $7 \mathrm{~kg}$ & $42.2 \mathrm{~cm}$ & Large \\
Butterfly Matchless & 3559 & 3 Years & 0.5 liter & $6 \mathrm{~kg}$ & $41.1 \mathrm{~cm}$ & Large \\
Pigeon Gusto & 1299 & 1 Year & $0.4 \mathrm{~L}$ & $2.7 \mathrm{~kg}$ & $27.99 \mathrm{~cm}$ & Small \\
\hline
\end{tabular}

Table 14. Precision, Recall and F1 calculation for Mixer-Grinder class.

\begin{tabular}{lcccccc}
\hline \multicolumn{7}{c}{ Product name: Mixer-grinder } \\
\hline $\begin{array}{l}\text { Attribute/instance } \\
\text { name }\end{array}$ & $\begin{array}{c}\text { Relevant docs } \\
\text { retrieved }\end{array}$ & $\begin{array}{c}\text { Total doc } \\
\text { retrieved }\end{array}$ & Precision & $\begin{array}{c}\text { Total } \\
\text { relevant doc }\end{array}$ & Recall & F1 \\
\hline Entity-name & 320 & 381 & 0.839 & 380 & 0.842 & 0.840 \\
Warranty & 200 & 228 & 0.877 & 333 & 0.600 & 0.712 \\
Capacity & 37 & 45 & 0.822 & 91 & 0.406 & 0.543 \\
Weight & 82 & 105 & 0.780 & 209 & 0.392 & 0.521 \\
Height & 68 & 78 & 0.871 & 107 & 0.635 & 0.734 \\
Size & 93 & 94 & 0.989 & 150 & 0.620 & 0.762 \\
Total & 800 & 931 & & 1270 & & \\
Average & & & 0.859 & & 0.629 & 0.726 \\
\hline
\end{tabular}

types of the attributes, maximum number of URLs crawled $(n)$ to limit the search space as 20 .

Output: $S P$ consisting of Television Size, Response Time, Weight, Weight, Angle, Size as Columns and rows of a $S P$ as their respective value-measure pairs. Sample $S P$ for Television is shown in Table 15 .

Table 15. Spreadsheet for television.

\begin{tabular}{lcccccc}
\hline Entity-name & Price & Television size & Response time & Weight & Angle & Size \\
\hline Panasonic & 15,699 & $32 \mathrm{inch}$ & 8 millisecond & $5 \mathrm{~kg}$ & 170 degrees & Large \\
Samsung & 11,659 & $18.5 \mathrm{inch}$ & $14 \mathrm{~ms}$ & $1.8 \mathrm{~kg}$ & 178 degrees & Big \\
Sony Bravia & 46,482 & $101.6 \mathrm{~cm}$ & 14 millisecond & $8.7 \mathrm{~kg}$ & 178 degrees & Large \\
LG & 18,490 & $80 \mathrm{~cm}$ & $12 \mathrm{~ms}$ & $6 \mathrm{~kg}$ & 175 degrees & Small \\
\hline
\end{tabular}

As mentioned in Table 16, we obtained 209 "Relevant Docs Retrieved", 218 "Total Doc Retrieved", precision as 0.958, "Total Relevant Doc" as 219, Recall as 0.995 and $\mathrm{F} 1$ as 0.976 for entity name. For Response time attribute, we obtained 76 "Relevant Docs Retrieved", 95 "Total Doc Retrieved", precision as 0.800, "Total Relevant Doc" as 101, Recall as 0.781 and F1 as 0.790 .

\subsubsection{Product class name: Electric timer}

Input: Search query specifying class name $C L N A M E$ as Electric Timer, attributes as Price, Timer size, Weight, Humidity, Current and Size, respective data types of the attributes, maximum number of URLs crawled $(n)$ to limit the search space as 20 . 
Table 16. Precision, recall and F1 calculation for television class.

\begin{tabular}{lcccccc}
\hline \multicolumn{7}{c}{ Product name: Television } \\
\hline Attribute/instance & $\begin{array}{c}\text { Relevant docs } \\
\text { retrieved }\end{array}$ & $\begin{array}{c}\text { Total doc } \\
\text { retrieved }\end{array}$ & Precision & $\begin{array}{c}\text { Total } \\
\text { relevant doc }\end{array}$ & Recall & F1 \\
\hline Entity-name & 209 & 218 & 0.958 & 219 & 0.995 & 0.976 \\
Television size & 143 & 178 & 0.803 & 191 & 0.748 & 0.774 \\
Response Time & 76 & 95 & 0.800 & 101 & 0.781 & 0.790 \\
Weight & 71 & 94 & 0.7551 & 101 & 0.702 & 0.727 \\
Angle & 53 & 60 & 0.883 & 62 & 0.854 & 0.868 \\
Size & 66 & 78 & 0.8461 & 100 & 0.66 & 0.741 \\
Total & 618 & 723 & & 774 & & \\
Average & & & 0.854 & & 0.798 & 0.825 \\
\hline
\end{tabular}

Output: $S P$ consisting of Timer size, Weight, Humidity, Current, Size as Columns and rows of a $S P$ as their respective value-measure pairs. Sample $S P$ for Electric Timer is shown in Table 17.

Table 17. Spreadsheet for electric timer.

\begin{tabular}{lcccccc}
\hline Entity-name & Price & Timer size & Weight & Humidity & Current & Size \\
\hline Walnut & 1598 & $17.8 \mathrm{~cm}$ & $50 \mathrm{gram}$ & $4585 \mathrm{rh}$ & $80 \mathrm{mah}$ & Small \\
Intermatic & $\$ 21.37$ & $16.6 \mathrm{~cm}$ & $46 \mathrm{~g}$ & $4565 \mathrm{rh}$ & $75 \mathrm{mah}$ & Small \\
Honeywell & $\$ 21.37$ & $18.6 \mathrm{~cm}$ & $51 \mathrm{~g}$ & $3898 \mathrm{rh}$ & $76 \mathrm{mah}$ & Small \\
Defiant & $\$ 24.97$ & $17.6 \mathrm{~cm}$ & $48 \mathrm{~g}$ & $4445 \mathrm{rh}$ & $78 \mathrm{mah}$ & Small \\
\hline
\end{tabular}

As mentioned in Table 18, we obtained 69 "Relevant Docs" Retrieved, 74 "Total Doc Retrieved", precision as 0.932, "Total Relevant Doc" as 80, Recall as 0.862 and F1 as 0.895 for entity name. For humidity attribute, authors obtained 5 "Relevant Docs Retrieved", 7 "Total Doc Retrieved", precision as 0.714, "Total Relevant Doc" as 13, Recall as 0.384 and F1 as 0.499 .

Table 18. Precision, recall and F1 calculation for electric timer class.

\begin{tabular}{lcccccc}
\hline \multicolumn{7}{c}{ Product name: Electric timer } \\
\hline $\begin{array}{l}\text { Attribute/instance } \\
\text { name }\end{array}$ & $\begin{array}{c}\text { Relevant } \\
\text { docs retrieved }\end{array}$ & $\begin{array}{c}\text { Total } \\
\text { doc retrieved }\end{array}$ & $\begin{array}{c}\text { Precision } \\
\text { relevant doc }\end{array}$ & Recall & F1 \\
\hline Entity-name & 69 & 74 & 0.932 & 80 & 0.862 & 0.895 \\
Timer size & 7 & 12 & 0.583 & 15 & 0.466 & 0.517 \\
Weight & 6 & 8 & 0.75 & 14 & 0.428 & 0.544 \\
Humidity & 5 & 7 & 0.714 & 13 & 0.384 & 0.499 \\
Current & 4 & 9 & 0.444 & 11 & 0.363 & 0.399 \\
Size & 12 & 12 & 1.0 & 17 & 0.705 & 0.826 \\
Total & 103 & 122 & & 150 & & \\
Average & & & 0.844 & & 0.686 & 0.756 \\
\hline
\end{tabular}

In the case of entity extraction, Table 19 shows the performance comparison of the proposed method with some of the prominent entity extraction methods 
Table 19. Comparison of proposed entity extraction method with the performance of methods in the literature.

\begin{tabular}{|c|c|c|c|c|c|}
\hline Dataset & Algorithm & Class & $\begin{array}{l}\text { Precision } \\
(\%)\end{array}$ & $\begin{array}{c}\text { Recall } \\
(\%)\end{array}$ & $\begin{array}{c}\text { F1-measure } \\
(\%)\end{array}$ \\
\hline Limited Web corpus & List Extractor (LE) [9] & $\begin{array}{l}\text { City } \\
\text { Film } \\
\text { Scientist }\end{array}$ & $\begin{array}{l}52 \\
72 \\
64\end{array}$ & $\begin{array}{l}\mathrm{NA}^{\mathrm{a}} \\
\mathrm{NA}^{\mathrm{a}} \\
\mathrm{NA}^{\mathrm{a}}\end{array}$ & $\begin{array}{l}\mathrm{NA}^{\mathrm{a}} \\
\mathrm{NA}^{\mathrm{a}} \\
\mathrm{NA}^{\mathrm{a}}\end{array}$ \\
\hline CONNL-2003 English & $\begin{array}{l}\text { Conditional Random } \\
\text { Fields (CRFs) [10] }\end{array}$ & $\begin{array}{l}\text { LOC } \\
\text { MISC }\end{array}$ & $\begin{array}{l}87.23 \\
74.44\end{array}$ & $\begin{array}{l}87.65 \\
71.37\end{array}$ & $\begin{array}{l}87.44 \\
72.87\end{array}$ \\
\hline Test Set & & $\begin{array}{l}\text { ORG } \\
\text { PER }\end{array}$ & $\begin{array}{l}79.52 \\
91.05\end{array}$ & $\begin{array}{l}78.33 \\
89.98\end{array}$ & $\begin{array}{l}78.92 \\
90.51\end{array}$ \\
\hline CONLL-2003 & $\begin{array}{l}\text { Context Pattern } \\
\text { Induction [11] }\end{array}$ & $\begin{array}{l}\text { Location } \\
\text { Organization } \\
\text { Person }\end{array}$ & $\begin{array}{l}70 \\
85 \\
88\end{array}$ & $\begin{array}{l}\mathrm{NA}^{\mathrm{a}} \\
\mathrm{NA}^{\mathrm{a}} \\
\mathrm{NA}^{\mathrm{a}}\end{array}$ & $\begin{array}{l}\mathrm{NA}^{\mathrm{a}} \\
\mathrm{NA}^{\mathrm{a}} \\
\mathrm{NA}^{\mathrm{a}}\end{array}$ \\
\hline CRL NE & $\begin{array}{l}\text { Support Vector Machine } \\
\quad(\mathrm{SVM})[12]\end{array}$ & $\begin{array}{l}\text { Artifact } \\
\text { Organization } \\
\text { Location } \\
\text { Person }\end{array}$ & $\begin{array}{l}\mathrm{NA}^{\mathrm{a}} \\
\mathrm{NA}^{\mathrm{a}} \\
\mathrm{NA}^{\mathrm{a}} \\
\mathrm{NA}^{\mathrm{a}}\end{array}$ & $\begin{array}{l}\mathrm{NA}^{\mathrm{a}} \\
\mathrm{NA}^{\mathrm{a}} \\
\mathrm{NA}^{\mathrm{a}} \\
\mathrm{NA}^{\mathrm{a}}\end{array}$ & $\begin{array}{l}50.16 \\
80.44 \\
88.57 \\
87.81\end{array}$ \\
\hline $\begin{array}{l}\text { All related web pages } \\
\text { returned by search } \\
\text { engine }\end{array}$ & Proposed algorithm & $\begin{array}{c}\text { Mobile Phone } \\
\text { Mixer Grinder } \\
\text { Television } \\
\text { Electric Timer }\end{array}$ & $\begin{array}{l}90.9 \\
83.9 \\
\mathbf{9 5 . 8} \\
\mathbf{9 3 . 2}\end{array}$ & $\begin{array}{l}\mathbf{9 1 . 2} \\
84.2 \\
\mathbf{9 9 . 5} \\
86.2\end{array}$ & $\begin{array}{c}\mathbf{9 1} \\
84 \\
\mathbf{9 7 . 6} \\
89.5\end{array}$ \\
\hline
\end{tabular}

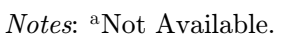

discussed in the literature. Table 20 shows the performance comparison of the proposed method with some of the prominent attribute-value extraction methods present in the literature. These methods have evaluated their performance on limited web corpus or a single site. We have evaluated the performance of

Table 20. Comparison of proposed value-measure extraction method with the performance of methods in the literature.

\begin{tabular}{|c|c|c|c|c|c|}
\hline Dataset & Algorithm & Class & $\begin{array}{l}\text { Precision } \\
\quad(\%)\end{array}$ & $\begin{array}{c}\text { Recall } \\
(\%)\end{array}$ & $\begin{array}{c}\text { F1-measure } \\
(\%)\end{array}$ \\
\hline $\begin{array}{l}\text { Single site } \\
\text { (www.dickssportinggoods. } \\
\quad \text { com) }\end{array}$ & $\begin{array}{l}\text { Naïve Bayes [17] } \\
\text { co-EM [17] }\end{array}$ & Football & $\begin{array}{l}31.85 \\
32.04\end{array}$ & $\begin{array}{l}45.38 \\
55.48\end{array}$ & $\begin{array}{l}\text { Not Available } \\
\text { Not Available }\end{array}$ \\
\hline $\begin{array}{l}\text { Only Tweets } \\
\text { Only Web-Links } \\
\text { Tweets + Web-Links }\end{array}$ & $\begin{array}{l}\text { POS tagging and } \\
\text { Dependency } \\
\text { Parsing [18] }\end{array}$ & $\begin{array}{c}\text { Tweets } \\
\text { Web-Links } \\
\text { Tweets }+ \text { Web-Links }\end{array}$ & $\begin{array}{l}85.1 \\
89.1 \\
87.4\end{array}$ & $\begin{array}{l}38.5 \\
29.3 \\
46.0\end{array}$ & $\begin{array}{l}51.6 \\
42.9 \\
59.5\end{array}$ \\
\hline $\begin{array}{l}\text { Single site } \\
\qquad \text { (www.rakuten.co.jp) }\end{array}$ & $\begin{array}{l}\text { Knowledge Base } \\
\text { Induction [19] }\end{array}$ & Wine and Shampoo & 57.05 & 59.66 & 58.15 \\
\hline $\begin{array}{l}\text { All related web } \\
\text { pages returned } \\
\text { by search engine }\end{array}$ & Proposed algorithm & $\begin{array}{l}\text { Mobile Phone } \\
\text { Mixer Grinder } \\
\text { Television } \\
\text { Electric Timer }\end{array}$ & $\begin{array}{l}73.75 \\
87.27 \\
80.99 \\
70.83\end{array}$ & $\begin{array}{l}55.49 \\
53.93 \\
\mathbf{7 3 . 6} \\
48.57\end{array}$ & $\begin{array}{c}63.33 \\
66.66 \\
77 \\
57.62\end{array}$ \\
\hline
\end{tabular}


the proposed method by crawling over almost every manufacturer or retailer websites.

The large improvement in the performance of the proposed algorithm for entity and value-measure extraction as compared with existing approaches (as shown in Tables 19 and 20) is due to application of POS tagging, generation of unique, enhanced, precise, important features and two classifications phased, one for entity extraction and other for attribute extraction. The authors have used the distance measure concept in order to extract the value-measure pair of the specified attribute and its data type. Due to the use of distance measure concept at the word level, extraction of value-measure pairs becomes very fast and accurate as compared with other methods discussed in the literature [17-19]. In [17], Naïve Bayes classifier has been used for classifying the word. The features used in this classifier are the surrounding 8 words of the particular word (unlabeled word) and their corresponding Parts Of Speech (POS) Tags. Naïve Bayes algorithm has to scan and work out on 8 words surrounding an unlabeled word. To improve the performance of Naïve Bayes, co-EM algorithm has been used in [17]. Co-EM is a semi-supervised algorithm, which utilizes 4 words before and 4 words after the particular word and their POS tags. Our proposed algorithm extracts only that word (either on the left side or right side of an attribute word) which is at the smallest distance as compared with attribute word. Due to this scenario, time complexity and accuracy of our proposed algorithm are better as compared with Naïve Bayes and co-EM.

A method for extracting numerical attribute-value pairs (which are mentioned side by side) in [18] is different as compared with the proposed algorithm. In [18], a word is marked as an attribute, if it is a noun and coming next to the word marked as numeric. This scenario is not applicable in ever cases. Consider the sentence, "2 MP camera". Using the method as described in [18], "MP" is marked an attribute for value "2" which is incorrect. Our proposed algorithm marks correctly "2 MP" as a value-measure pair for "camera" attribute. This indicates that accuracy (F1-measure) of our proposed algorithm is more as compared with the technique used in [18] which is shown in Table 20. The accuracy (F1-measure) of the model which uses Knowledge Base (KB) [19] for extracting attribute-value pairs are also less as compared with the proposed algorithm as shown in Table 20.

The authors have also used the distance measure concept as well as "Section" feature to efficiently extract entity name for a particular class. Due to the utilization of "Section" feature, instead of searching complete web page, our proposed algorithm searches only the initial part of a web page which results in fast extraction of entity name as compared with other methods discussed in the literature [9-12]. As shown in Table 19, [9] utilizes List Extraction (LE) method, which initially discovers an exact place of lists of class instances on the web page. Then, for each list, a wrapper is learned and finally, by utilizing these wrappers list elements are extracted. This wrapper induction (rule extraction) technique will not work always because, on each web page, an arrangement of entity names is done in a different fashion. In [10], CRFs are used which utilizes Conditional Probability for extracting entity names. 
Unlike our method, this method requires a large amount of hand-labeled training data. In this method, experimentation was performed on only one data set, i.e. "CONLL-2003 English Test Set". We have performed experimentation on almost all related web pages returned by a search engine. The technique described in [11], automatically extracts entity dictionaries from a large amount of text. However, this method requires complicated induction patterns to extract entity names. Technique addressed in [12] is based on SVM which also requires a large dataset manually labeled for training.

F1-Measure of our proposed algorithm is maximum among all the other models $[10,12,18,19]$. The higher value of F1-measure represents that our proposed algorithm is more accurate than existing models studied in the literature. The precision of our proposed algorithm is maximum among all other models [9, 11, 17, 19]. The higher value of precision indicates that the proposed algorithm is more robust to remove the unimportant information from the text. A Higher value of recall of the proposed algorithm as compared with $[17,18]$ indicates that the proposed algorithm is robust to keep the important information from the text.

Works which is described in $[13,15,16]$ are different than proposed work which described in this paper in such a way that, in proposed work authors extract valuemeasure pairs given their attributes and data types. The authors also execute the extraction process on product description pages of different retailers. These pages are highly unstructured as compared to online reviews. Attribute names are often different in online reviews as compared with the retailers product description page. For example, in online reviews, customer mentions picture quality as an attribute, but in the product description, page retailer will mention it as camera as an attribute.

\section{Conclusion and Future Work}

A big amount of data related to a particular class of product is available online in different web pages. Single retailer site can not satisfy the customer, as it has limited information regarding particular product attributes. Prices of the product may also vary from one site to another. A lot of time will be saved if all the information regarding a particular class of products is consolidated in the spreadsheet. The major challenge to provide such consolidated information is to extract this information from unstructured text using Natural Language Processing.

In the proposed work, the authors have come up with a novel idea of generation of web-spreadsheet for the specified class name. A classifier-based technique for entity extraction and attribute value extraction is also suggested. A classifier is trained using product classes of different categories, which experimentally shows that the classifier built using features can be used for any product classes without the need of trained data of each class.

In the proposed work, nearly every websites of retailers have been crawled. These sites are basically a result returned by search engine for a particular class query 
formed by user. Proposed novel algorithm efficiently extracts the entity name and value-measure pairs for related web pages and generates one row for corresponding web page Di. Separate J48 decision tree classifier is used to extract the entity name and value-measure pairs. Authors have used various product classes for generating training data for both the classifiers. Classifier designed for entity, efficiently classifies words from test documents as entity name and the other classifier which is designed for value-measure pair, efficiently classifies words from test documents as value-measure pair. In this work, authors have dealt with attributes, whose value measure pairs belongs to Alphanumeric, Numeric-Alpha and String category.

Experiments were rigorously performed on Mobile-Phone, Tablet, Mixer-Grinder, Television, Washing Machine, Tennis Racket and Electric Timer product classes and promising results are obtained in each category. Tables 19 and 20 show that the proposed method accuracy is superior to many entity and attribute-value extraction techniques.

Immediate future work is to automatically extract attribute and value-measure pairs from unstructured web documents for a particular product class and generate spreadsheet for the same.

\section{References}

1. A. Kopliku, K. Pinel-Sauvagnat and M. Boughanem, Aggregated search: A new information retrieval paradigm, ACM Comput. Surv. 46(3) (2014) 41.

2. J. Turmo, A. Ageno and N. Catal, Adaptive information extraction, ACM Comput. Surv. $\mathbf{3 8}(2)(2006) 4$.

3. O. Etzioni, A. Fader, J. Christensen, S. Soderland and M. Mausam, Open information extraction: The second generation, IJCAI 11 (2011) 3-10.

4. J. Wang, C. Chen, C. Wang, J. Pei, J. Bu, Z. Guan and W. V. Zhang, Can we learn a template-independent wrapper for news article extraction from a single training site? in Proc. 15th ACM SIGKDD Int. Conf. Knowledge Discovery and Data Mining, 2009, pp. 1345-1354.

5. P. Nesi, G. Pantaleo and M. Tenti, Ge (o) lo (cator): Geographic information extraction from unstructured text data and web documents, in 9th IEEE Int. Workshop on Semantic and Social Media Adaptation and Personalization, 2014, pp. 60-65.

6. C. H. Chang, M. Kayed, M. R. Girgis and K. F. Shaalan, A survey of web information extraction systems, IEEE Trans. Knowl. Data Eng. 18(10) (2006) 1411-1428.

7. X. Wu, T. W. Ling, M. L. Lee and G. Dobbie, Designing semistructured databases using ORA-SS model, in Proc. Second Int. Conf. on Web Information Systems Engineering, 2001, pp. 171-180.

8. X. Zhang, P. Lv, C. Zhao and J. Wang, A method for materials knowledge extraction from HTML tables based on sibling comparison, Int. J. Softw. Eng. Knowl. Eng. 26(6) (2016) 897-926.

9. O. Etzioni, M. Cafarella, D. Downey, A. M. Popescu, T. Shaked, S. Soderland, D. S. Weld and A. Yates, Unsupervised named-entity extraction from the web: An experimental study, Artif. Intell. 165(1) (2005) 91-134. 
10. A. McCallum and W. Li, Early results for named entity recognition with conditional random fields, feature induction and web-enhanced lexicons, in Proc. Seventh Association for Computational Linguistics Conf. Natural Language Learning, 2003, pp. 188-191.

11. P. P. Talukdar, T. Brants, M. Liberman and F. Pereira, A context pattern induction method for named entity extraction, in Proc. Tenth Association for Computational Linguistics Conf. Computational Natural Language Learning, 2006, pp. 141-148.

12. M. Asahara and Y. Matsumoto, Japanese named entity extraction with redundant morphological analysis, in Proc. 2003 Association for Computational Linguistics Conf. North American Chapter of the Association for Computational Linguistics on Human Language Technology, 2003, pp. 8-15.

13. J. Jin, P. Ji and R. Gu, Identifying comparative customer requirements from product online reviews for competitor analysis, Eng. Appl. Artif. Intell. 49 (2016) 61-73.

14. M. Hu and B. Liu, Mining opinion features in customer reviews, in AAAI, 2004, pp. $755-760$.

15. A. M. Popescu and O. Etzioni, Extracting product features and opinions from reviews, in Natural Language Processing and Text Mining (Springer, London, 2007), pp. 9-28.

16. M. Hu and B. Liu, Mining and summarizing customer reviews, in Proc. Tenth ACM SIGKDD Int. Conf. Knowledge Discovery and Data Mining, 2004, pp. 168-177.

17. R. Ghani, K. Probst, Y. Liu, M. Krema and A. Fano, Text mining for product attribute extraction, ACM SIGKDD Explor. Newslett. 8(1) (2006) 41-48.

18. S. Panem, M. Gupta and V. Varma, Structured information extraction from natural disaster events on twitter, in Proc. 5th ACM Int. Workshop on Web-Scale Knowledge Representation Retrieval \& Reasoning, 2014, pp. 1-8.

19. K. Shinzato and S. Sekine, Unsupervised extraction of attributes and their values from product description, in IJCNLP, 2013, pp. 1339-1347.

20. T. Joachims, Text categorization with support vector machines: Learning with many relevant features, in Proc. 10th European Conf. Machine Learning, Chemnitz, Germany, 1998, pp. 137-142.

21. F. Sebastiani, Machine learning in automated text categorization, ACM Comput. Surv. 34(1) (2002) 1-47.

22. Y. Yang and X. Liu, A re-examination of text categorization methods, in Proc. 22nd Annual Int. ACM SIGIR Conf. Research and Development in Information Retrieval, 1999, pp. 42-49.

23. J. R. Quinlan, C4. 5: Programs for Machine Learning (Elsevier, 2014).

24. B. Hssina, A. Merbouha, H. Ezzikouri and M. Erritali, A comparative study of decision tree ID3 and C4. 5, Int. J. Adv. Comput. Sci. Appl. 4(2) (2014) 13-19. 